\title{
Dispersive waves in fs cascaded second-harmonic generation
}

Morten Bache ${ }^{1}$, Ole Bang ${ }^{1}$, Wieslaw Krolikowski ${ }^{1,2}$ and Frank W. Wise ${ }^{3}$

1. DTU Fotonik, Department of Photonics Engineering, Technical University of Denmark, DK-2800 Kgs. Lyngby, Denmark

2. Research School of Physical Sciences and Engineering, Australian National University, Canberra ACT 0200, Australia

3. Department of Applied and Engineering Physics, Cornell University, Ithaca, New York 14853, USA

Optical solitons are nondispersive nonlinear waves that are supported through a balance between group-velocity dispersion (GVD) and nonlinearity. The so-called dispersive waves are linear waves phase-matched to these solitons, and are often found in supercontimuum generation [1,2]. The dispersive wave wavelength can be predicted from a phase-matching condition to the solitonic wave [3]. This phase-matching point is often interpreted as being located where solitons are no longer supported, i.e. where the GVD changes sign. However, in reality the dispersive wave is found at the wavelength where the total dispersion of the generated soliton is changing sign.

Recently, we studied cascaded second-harmonic generation (SHG) for soliton compression of fs pulses and found numerical evidence of dispersive-wave formation [4]. For the setup used (a $\beta$-barium borate, BBO, nonlinear crystal set up for type I SHG pumped at $\lambda_{1}=1.06 \mu \mathrm{m}$ ) the dispersive waves were strongly red-shifted compared to the compressed ultra-short near-IR soliton, and this probably explains why they have not been observed before. We noted that they appeared around the wavelength where the total dispersion of the pump wave changed sign.

Here we study this phenomenon in further detail by modelling an intense fs pulse propagating in a quadratic nonlinear crystal, where phase mismatched (cascaded) SHG takes place. As the pump pulse propagates, a huge nonlinear phase shift - and thus a chirp - is created on it due to cascaded SHG. In presence of proper dispersion this strongly chirped pulse can through the soliton effect be compressed using to a duration of just a few cycles $[4,5]$. The physics behind this is that the pump pulse may propagate as a Kerr-like soliton: if the cascaded SHG process has a negative phase mismatch $\Delta k=k_{2}-2 k_{1}<0$, where $k_{j}=n_{j}(\omega) \omega / c$, then a self-focusing Kerr-like nonlinearity is created and solitons are therefore supported with anomalous GVD. However, if $\Delta k>0$ the pump feels the cascaded SHG as a self-defocusing Kerr-like nonlinearity and solitons are supported with normal GVD.
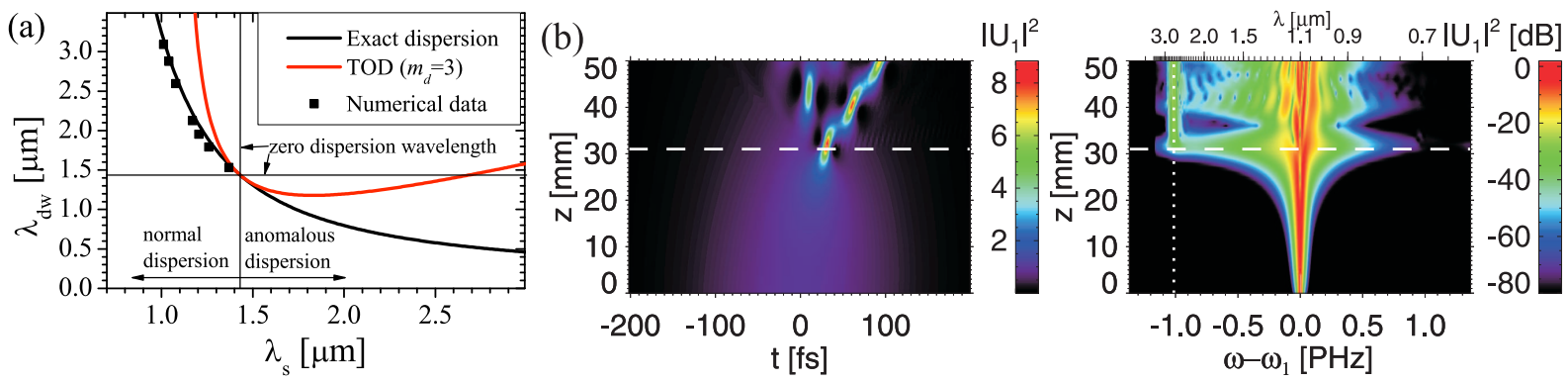

Fig. 1. (a) The calculated phase-matching curve showing the wavelength of the dispersive wave $\lambda_{\mathrm{dw}}$ vs. the soliton $\lambda_{\mathrm{s}}$ in a BBO nonlinear crystal. The effect of including only up to 3rd order dispersion is also shown. (b) Numerical simulation $\left(\lambda_{1}=1.1 \mu \mathrm{m}, T_{\text {in }}=200 \mathrm{fs}\right.$ FWHM, $I_{\text {in }}=78 \mathrm{GW} / \mathrm{cm}^{2}$ and $\left.\Delta k=60 \mathrm{~mm}^{-1}\right)$ : the pulse compresses to $7.0 \mathrm{fs}$ at the dashed line, and a dispersive wave appears close to the predicted wavelength (dotted line). The result of this and other numerical simulations with $\lambda_{1} \in[1.03-1.40] \mu \mathrm{m}$ are shown in (a).

A dispersive wave can get phase matched to the compressed soliton at a wavelength determined by the zero point in frequency domain of the dispersion operator for the fundamental wave: $\sum_{m=2}^{m_{d}} \frac{\left(\omega-\omega_{s}\right)^{m}}{m !}\left(\frac{\partial^{m} k_{1}}{\partial \omega^{m}}\right)_{\omega=\omega_{s}}$, where $m_{d}$ is the dispersion order. We plot this phase-matching condition for exact dispersion $\left(m_{d}=\infty\right)$ and 3 rd order dispersion $\left(m_{d}=3\right)$ in Fig. 1(a). It is evidently crucial to use exact dispersion. We also show the results of numerical simulations of a plane-wave model [4] with exact dispersion. We found dispersive waves phase-matched to the compressed few-cycle solitons with normal GVD $\left(\lambda_{1}<1.45 \mu \mathrm{m}\right)$. These results confirm the analytical calculations. A simulation example is shown in (b), where a $\lambda_{1}=1.1 \mu \mathrm{m}$ few-cycle soliton is generated after propagating $30 \mathrm{~mm}$ and then a dispersive wave appears close to the predicted $\lambda_{\mathrm{dw}} \simeq 2.70 \mu \mathrm{m}$. For $\lambda_{1}>1.45 \mu \mathrm{m}$ the GVD of BBO is anomalous and solitons are therefore of the self-focusing kind. Such solitons would show highly distorted beam profiles due to self-focusing effects, and cannot be described by our plane-wave model.

\section{References}

[1] J. Herrmann et al., "Experimental Evidence for Supercontinuum Generation by Fission of Higher-Order Solitons in Photonic Fibers," Phys. Rev. Lett. 88, 173901 (2002).

[2] D. V. Skryabin et al., "Soliton Self-Frequency Shift Cancellation in Photonic Crystal Fibers," Science 301, 1705-1708 (2003).

[3] N. Akhmediev and M. Karlsson, "Cherenkov radiation emitted by solitons in optical fibers," Phys. Rev. A 51, 2602-2607 (1995).

[4] M. Bache et al., "Limits to compression with cascaded quadratic soliton compressors," Opt. Express 16, 3273-3287 (2008).

[5] J. Moses and F. W. Wise, "Soliton compression in quadratic media: high-energy few-cycle pulses with a frequency-doubling crystal," Opt. Lett. 31, 1881-1883 (2006). 


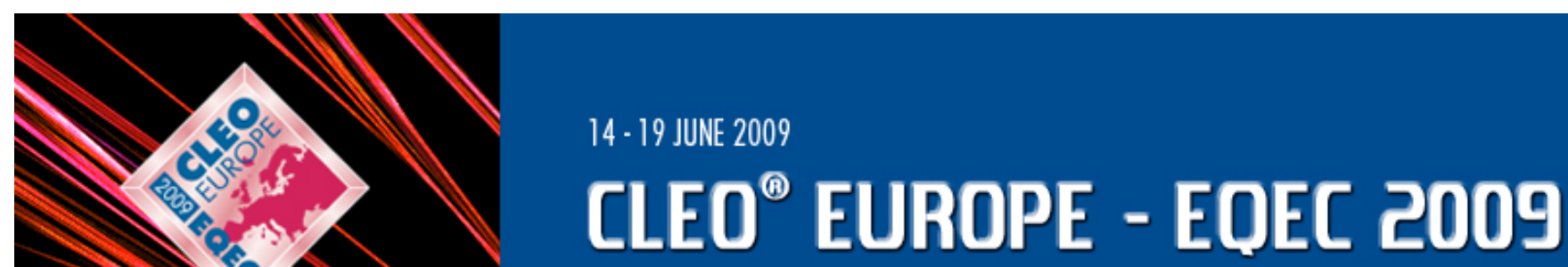

CONFERENCE ON LASERS AND ELECTRO-OPTICS - EUROPEAN QUANTUM ELECTRONICS CONFERENCE

\section{GENERAL CONFERENCE}

\section{Online Submissions}

Summary Information

This page provides practical inforr deadline is Monday 26 January 2

If you have previously read the $d \epsilon$ http://cleo09.sciconf.org/ Pleas $\epsilon$

Detailed Paper Preparation

\section{veaunIes}

SHORT

COURSES

REGISTER

PROGRAM

\section{Topics}

Paper Submission

Postdeadline Submission

ers to CLEO®/Europe 2009 and EQEC 2009. The submission

Is, you can directly access the paper submission site at : opy of the copyright form as described below.

Detailed Paper Preparation пाоничимо

A number of contributed papers covering original, unpublished work on the conference topics will be accepted for presentation. Papers should be submitted to only one conference. The CLEO®/Europe 2009 and EQEC 2009 programme committees will transfer papers from one conference to the other where appropriate unless written instructions to the contrary are given by the author at the time of submission.

Online submissions are now open

The deadline for submission of the papers is Monday 26 January 2009.

Submissions will be accepted up until midnight Monday French time. That is: 23h00 (11 pm) GMT Monday 26 January or $18 \mathrm{~h} 00$ (6 pm) EST Monday 26 January.

\section{Absolutely no papers will be accepted after this deadline.}

Authors will be notified whether their papers have been accepted by 16 March 2009.

Registration is open to all members of the scientific and technical community. Authors must obtain appropriate approval to have their paper reviewed by and presented to an international audience.

\section{Two types of contribution can be chosen: \\ $\rightarrow$ Oral or Poster \\ -> Poster only}

\section{The first option will automatically be entered unless you change it.}

Choosing the first option (Oral or Poster) will mean that your submission is automatically submitted as an oral contribution to the refereeing process. However, the programme committee can decide to affect it as a poster.

Choosing the second option (Poster only) will mean that your submission is only eligible for a poster presentation.

The option "Invited Talk" is only authorized for the pre-invited presenters. Do not choose this option unless you were explicitly formerly pre-invited to present an invited talk at the Conference.

Authors are requested to:

1) Electronically submit their paper (a 35-word abstract and a one page summary in pdf format) via http://cleo09.sciconf.org/

2) Email a copy of the copyright form (.pdf or .doc) to the EPS office at conferences@eps.org or directly send it via the on line submission system

Required documents:

\section{A 35-word abstract}

2. A one page summary in pdf format (download template: .doc | .pdf)

Please follow the following layout recommendations

- Paper size: A4 format $(210 \mathrm{~mm} \times 297 \mathrm{~mm})$

- Margins: left \& right $=25 \mathrm{~mm}$, top \& bottom $=25 \mathrm{~mm}$

- Format: Acrobat ( ${ }^{\star}$.pdf) file

- Title: use 14pt Times bold letters centred on the page, elements and acronyms should be capitalized.

- list all authors' names, organisation/affiliation \& mailing address under the title:

Centre author name(s) on one line. Use 10-point, bold font. Author names should include first and middle names or initials followed by surname. Their affiliations must be indicated through the use of appropriate superscripts. 
Centre author affiliation(s) on the following lines. Use 8-point, italic font. Affiliations should follow the format: division, organization, address. Do not use abbreviations.

\section{- Introductory Text}

Please be concise in your presentation, highlighting what is novel and original about your submission. Do not repeat the separate 50 word abstract.

- Main text

The first line of the first paragraph of a section or subsection should start flush left. The first line of subsequent paragraphs within the section or subsection should be indented $0.5 \mathrm{~cm}$.

\section{- Tables}

Tables should be centred and numbered consecutively. Table titles should be centred above the table and in a 10-point font. If the title is more than one line, it should have margins that match the width of the table. Tables should be positioned as close as possible to where they are mentioned in the main text.

\section{- Figures}

Figures should also be placed as close as possible to where they are mentioned in the text. All the figures should be centred, except for small figures less than $6 \mathrm{~cm}$ in width, which may be placed side by side. No part of a figure should extend beyond the typing area. Text should not wrap around figures. The figures should be provided in greyscale.

Figure captions should be centred beneath figures and in an 8-point font. Figure captions should be indented $1 \mathrm{~cm}$ on both sides and justified on both right and left sides.

\section{- Equations}

Equations should be centred, unless they are so long that less than $1 \mathrm{~cm}$ will be left between the end of the equation and the equation number, in which case they may run on to the next line. Equation numbers should only appear to the right of the last line of the equation, in parentheses. For long equations, the equation number may appear on the next line. For very long equations, the right side of the equation should be broken into approximately equal parts and aligned to the right of the equal sign.

\section{- References}

References should appear at the end of the article in the order in which they are referenced in the body of the paper. The font should be 8 point, and the references should be aligned left.

Within the main text, references should be designated by a number in brackets [1], and they should precede a comma or period [2].

Two references cited at once should be included together [2,3], separated by a comma, while three or more consecutive references should be indicated by the bounding numbers and a dash [1-3].

For journal articles, authors are listed first, followed by the article's full title in quotes, the journal's title abbreviation, the volume number in bold, page number, and the year in parentheses.

[1] J. Itatani, D. Zeidler, J. Levesque, D. M. Villeneuve, and P. B. Corkum, "Controlling High Harmonic Generation with Molecular Wave Packets," Phys. Rev. Lett. 94, 123902 (2005).

For citation of a book as a whole: authors, followed by title in italics, and publisher, city, and year in parenthesis.

[2] G. P. Agrawal, Nonlinear Fiber Optics, 3rd. ed., (Academic Press, Boston, 2001).

For citation of a book chapter, authors are listed first, followed by book title in italics, editors, and publisher, city, and year in parenthesis. Chapter number may be added if applicable.

[3] R. Kienberger and F. Krausz in, Few-cycle laser pulse generation and its applications, F.X. Kärtner ed. (Springer Verlag, Berlin, 2004).

For citation of proceedings, follow the individual proceedings format:

[4] Author(s), "Title of paper," in Title of Proceeding, Ed(s) Name(s), Proc. format ......

\section{- Miscellaneous}

Acknowledgments, job descriptions and footnotes should not be included in the summary.

Do not add any page number. Do not use hyphens at the end of a line.

Directives for the electronic submission:

The submission procedure takes four steps. Besides filling the online form you need to upload a one page abstract in PDF format Your contribution is properly submitted after performing all the steps only. You will get a proper success message online and by email.

The online submission system properly handles numerous special characters. Details are available at the information page on special characters. Optionally you can also create mathematical symbols and formulas via LaTeX. Unfortunately some characters will get a special meaning once LaTeX is activated and the characters need to be replaced by proper code. In case of problems with the online submission system technical support is available at contact@wobsta.de

Please take care of the following hints:

- Do not use CAPITAL WORDS for example in author names or the title (except for common acronyms).

Examples:

Do not write TRANSPORT AND OPTICS IN HIGH MAGNETIC FIELDS but Transport and optics in high magnetic fields. Do not write PETER SMITH but Peter Smith

Do not write EUROPEAN PHYSICAL SOCIETY, Mulhouse, FRANCE but European Physical Society, Mulhouse, France However write C.N.R.S.

- Be sure to not exchange the first name and the surname. Otherwise you will be listed by your first name in the author index.

- Prefer to use full first names. Middle initials can be added after the first name. Do not state academic titles.

- Submit all the authors' information and add as many authors as required.

The system will not allow the conference organiser to make any change and the person registering the submission is entirely responsible for entering the full and correct list of all the authors. 
- Do not misuse the LaTeX functionality to alter the font. However it's acceptable to use single italic words or the usual bold notation for volume numbers in citations. Do not create empty vertical space. Do not use "II" to create line breaks.

- You need to upload a one page summary in PDF format. This extended version of the abstract will be burnt on a CD to be included in the conference digest.

\section{Alternative 1: Restore an already filled form}

It is possible to fill out a contribution form and to create a format test but instead of submitting the contribution right away you can download your data in form of a LaTeX file. Using this file you can then later on restore the filled form.

\section{Alternative 2: Modify an already submitted abstract}

In the exceptional case an already submitted contribution needs to be modified, please fill in the key for modification you received during the contribution submission.

In the final step you will receive a submission confirmation on screen and by email. Be sure to get this confirmation to ensure the proper registration of your contribution in the conference database. The confirmation email which will be sent will contain the format test as pdf for your records and a key for corrections. Corrections are authorized until 11 May 2009 . After that deadline the review process will begin and no change can any longer be brought.

These recommendations are intended to avoid technical problems in the transferral of your paper to the conference digest. Failure to follow these recommendations may result in papers being returned to authors. Please note that the EPS will not manipulate or edit papers.

The summary will be reduced and published in the Technical Digest. Since contributed papers are selected on the basis of the summary, it should convey the original results in a succinct manner rather than describe the research topic.

\section{Any of the following conditions may result in rejection of a paper:}

- failure to submit the paper by the deadline date

- failure to complete the required fields on the web based submission form

- failure to follow the compulsory layout recommendations (a short abstract, a one page summary in pdf format)

- failure to send the copyright form.

The date and time for presentation will be determined after the programme committee has reviewed the papers.

Authors will be notified whether their papers have been accepted by 16 March 2009. Notifications will be sent to the email address as given during the electronic submission. 